\title{
SIMILARITY REDUCTION OF ENERGY-TRANSPORT MODELS FOR SEMICONDUCTORS
}

\author{
M Molati and C Wafo Soh \\ School of Computational and Applied Mathematics \\ Centre for Differential Equations, Continuum Mechanics and Applications \\ University of the Witwatersrand, Private Bag 3, Wits 2050, \\ Johannesburg, South Africa \\ m.molati@nul.ls andwafo@cam.wits.ac.za
}

\begin{abstract}
The main purpose of this work is to perform symmetry classification of a system of partial differential equations for energy-transport in semiconductors. In the case where there are symmetries, they are used to reduce the number of independent variables which in turn enables one to construct invariant solutions. Invariant solutions of a given equation satisfy an equation with fewer independent variables. Thus, the search of invariant solutions can be viewed as a sort of dimensional reduction. From a computational standpoint, the reduced system is easier to analyse both numerically and analytically than the original system.
\end{abstract}

Key Words- Energy-transport, symmetry, invariant solution, dimensional reduction

\section{INTRODUTION}

The motion of charge carriers (negatively charged particles, electrons and positively charged particles, holes) in semiconductors under the effect of an electric field and a carrier concentration gradient is an important phenomenon. This introduces an important parameter, mobility, which characterizes the motion of the charge carriers due to drift (charged-particle motion under the influence of the electric field) and also the efficiency of many devices. The numerical value of the mobility depends on a given doping (addition of controlled amounts of specific impurity atoms with the aim of increasing the concentration of the charge carriers) and temperature for the charge carriers $[9,11]$.

The energy-transport (ET) model is a macroscopic model derived from Boltzmann equation [1]. This model comprises a system of diffusion equations for the electron density and temperature, together with the Poisson equation for electric potential.

In the ET model for semiconductors, there are two groups of unknowns. The first group is made of the electron density, the electron temperature and the electric potential. The second group is formed by the energy production, the mobilities and the doping profile. Traditionally, the latter is obtained experimentally. This work employs the symmetry principle $[2,5,7,8]$ to get the elements of the second group of the unknowns, that is, the forms of the energy production, the mobilities and the doping profile are obtained for which the model is maximally symmetric. Symmetries of a system of partial differential equations (PDEs) for the ET model will be calculated from the so-called determining equations (an overdetermined system of linear homogeneous PDEs). 
When the original system contains arbitrary parameters or functions, the consistency conditions of the determining equations provides a means to specify their forms. This is the essence of the group classification method [8]. The importance of group classification stems from the fact that many models in application contain parameters or functions which cannot be determined from any known physical law. However, by analysing the consistency conditions of the determining equations we are able to identify maximally symmetric submodels.

In some problems of practical interest, generating the determining equations turns out to be tedious. Fortunately, Lie's method for calculating symmetries is algorithmical and can be implemented using packages for symbolic computation (Mathematica, Maple, Reduce, ...). In this work we will use the program, Yalie [4], written in Mathematica for the generation and manipulation of the determining equations for symmetries.

The outline of this paper is as follows. In section 2, we present the model to be investigated. In section 3, we perform the symmetry classification of the model. In section 4, we employ the symmetries to optimally reduce the number of independent variables in the model. Finally, we summarize our findings and hints on further work.

\section{ENERGY-TRANSPORT MODEL FOR SEMICONDUCTORS}

The detailed derivation of the energy-transport (ET) model from the Boltzmann equation is presented in [1]. On coupling the Poisson equation for the electric potential to the diffusion equations for the electron density and temperature, we have the following equations [10]

$$
\begin{aligned}
\frac{\partial \mathrm{n}}{\partial \mathrm{t}}+\nabla \cdot \mathbf{J} & =0, \\
\frac{\partial(\mathrm{nW})}{\partial \mathrm{t}}+\nabla \cdot \mathbf{S}-\mathbf{J} \cdot \nabla \varphi-\mathrm{nC}_{\mathrm{w}} & =0 \\
\lambda^{2} \nabla^{2} \varphi-\mathrm{n}+\mathrm{c}(\mathrm{x}) & =0,
\end{aligned}
$$

where $\mathrm{n}$ is the electron density, $\mathbf{J}$ the electron momentum density, $\mathrm{W}$ the electron energy, $\mathbf{S}$ the energy flux density, $\mathrm{nC}_{\mathrm{W}}$ the energy production, $\lambda^{2}$ the dielectric constant, $\varphi$ the electric potential, $\mathrm{c}(\mathrm{x})$ the doping profile and $\nabla=\left(\frac{\partial}{\partial \mathrm{x}}, \frac{\partial}{\partial \mathrm{y}}, \frac{\partial}{\partial \mathrm{z}}\right)$. The known quantities in (1) are

$$
\mathbf{J}=-\nabla\left(\mu^{(1)} \mathrm{Tn}\right)+\mu^{(1)} n \nabla \varphi, \mathbf{S}=-\nabla\left(\mu^{(2)} \mathrm{T}^{2} \mathrm{n}\right)+\mu^{(2)} \mathrm{Tn} \nabla \varphi, \mathrm{C}_{\mathrm{w}}=\frac{-\frac{3}{2}\left(\mathrm{~T}-\mathrm{T}_{\mathrm{L}}\right)}{\tau_{\mathrm{w}}(\mathrm{T})}, \mathrm{W}=\frac{3}{2} \mathrm{~T},
$$

where $\mathrm{T}$ is the electron temperature, $\mathrm{T}_{\mathrm{L}}$ is the lattice temperature (taken as constant), $\mu^{(\mathrm{i})}$ are the electron mobilities and $\tau_{\mathrm{W}}$ is the energy relaxation time. In general the mobilities are temperature-dependent. The system (1) must be solved subject to appropriate initial and boundary conditions.

Some special cases recently considered in the literature are:

- the Chen et al [3] model with 
$\mathbf{J}=-\mu_{0}\left[\nabla \mathrm{n}-\frac{\mathrm{n}}{\mathrm{T}} \nabla \varphi\right], \quad \mathbf{S}=-\frac{3}{2} \mu_{0}[\nabla(\mathrm{nT})-\mathrm{n} \nabla \varphi]$ and $\mathrm{C}_{\mathrm{w}}=\frac{-\frac{3}{2}\left(\mathrm{~T}-\mathrm{T}_{\mathrm{L}}\right)}{\tau_{0}}$,

where $\mu_{0}$ and $\tau_{0}$ are positive constants,

- the Lyumkis et al [6] model in which

$\mathbf{J}=-\frac{2 \mu_{0}}{\sqrt{\pi}}\left[\nabla\left(\mathrm{nT}^{\frac{1}{2}}\right)-\frac{\mathrm{n}}{\mathrm{T}^{\frac{1}{2}}} \nabla \varphi\right], \mathbf{S}=-\frac{4 \mu_{0}}{\sqrt{\pi}}\left[\nabla\left(\mathrm{nT}^{\frac{3}{2}}\right)-\mathrm{nT}^{\frac{1}{2}} \nabla \varphi\right]$ and $\mathrm{C}_{\mathrm{w}}=-\frac{2}{\sqrt{\pi}} \frac{\left(\mathrm{T}-\mathrm{T}_{\mathrm{L}}\right)}{\tau_{0} \mathrm{~T}^{\frac{1}{2}}}$.

\section{THE SYMMETRY CLASSIFICATION OF THE ET MODEL FOR SEMICONDUCTORS}

Recently Romano and Valenti [10] performed the symmetry analysis of the one-dimensional ET model for semiconductors. They also calculated invariant solutions. In this section we will continue their work by examining multidimensional models, namely, we perform the group classification of the spherically symmetric and the two-dimensional ET models for semiconductors. In order to generate and manipulate the determining equations for symmetries, we use the Mathematica software Yalie developed by Díaz [4].

\subsection{The spherically symmetric ET model for semiconductors}

Here we focus on the symmetry analysis of the spherically symmetric ET model for semiconductors. By spherically symmetric we mean that all spatial dependence of the dependent variables is through the radial coordinate

$$
r=\sqrt{\left(x^{1}\right)^{2}+\left(x^{2}\right)^{2}+\cdots\left(x^{d}\right)^{2}}
$$

where $\mathrm{d}$ is the spatial dimension. Using the chain rule, we arrive at the following spherically symmetric equations

$$
\begin{array}{r}
\mathrm{n}_{\mathrm{t}}+\mathrm{J}_{\mathrm{r}}+\frac{\mathrm{k}}{\mathrm{r}} \mathrm{J}=0, \\
\frac{3}{2}(\mathrm{nT})_{\mathrm{t}}+\mathrm{S}_{\mathrm{r}}+\frac{\mathrm{k}}{\mathrm{r}} \mathrm{S}-\mathrm{J} \varphi_{\mathrm{r}}+\frac{3}{2} \mathrm{n} \frac{\left(\mathrm{T}-\mathrm{T}_{\mathrm{L}}\right)}{\tau_{\mathrm{w}}(\mathrm{T})}=0, \\
\lambda^{2}\left(\varphi_{\mathrm{rr}}+\frac{\mathrm{k}}{\mathrm{r}} \varphi_{\mathrm{r}}\right)-\mathrm{n}+\mathrm{c}(\mathrm{r})=0,
\end{array}
$$

where the subscript denotes partial differentiation, $k=d-1, J=-\left(\mu^{(1)} T n\right)_{r}+\mu^{(1)} n \varphi_{r}$ and $S=-\left(\mu^{(2)} T^{2} n\right)_{r}+\mu^{(2)} \operatorname{Tn} \varphi_{r}$. The cases of interest are $d=1,2,3$, i.e. $k=0,1,2$.

From now on we will use $\tau(\mathrm{T})$ to mean $\tau_{\mathrm{W}}(\mathrm{T})$. According to Lie's algorithm, the vector field

$$
\begin{aligned}
X= & \xi^{1}(\mathrm{t}, \mathrm{r}, \mathrm{n}, \mathrm{T}, \varphi) \frac{\partial}{\partial \mathrm{t}}+\xi^{2}(\mathrm{t}, \mathrm{r}, \mathrm{n}, \mathrm{T}, \varphi) \frac{\partial}{\partial \mathrm{r}}+\eta^{1}(\mathrm{t}, \mathrm{r}, \mathrm{n}, \mathrm{T}, \varphi) \frac{\partial}{\partial \mathrm{n}} \\
& +\eta^{2}(\mathrm{t}, \mathrm{r}, \mathrm{n}, \mathrm{T}, \varphi) \frac{\partial}{\partial \mathrm{T}}+\eta^{3}(\mathrm{t}, \mathrm{r}, \mathrm{n}, \mathrm{T}, \varphi) \frac{\partial}{\partial \varphi}
\end{aligned}
$$

is a symmetry generator of (2) if 


$$
\begin{aligned}
X^{[2]}\left(n_{t}+J_{r}+\frac{k}{r} J\right) & =0, \\
X^{[2]}\left(\frac{3}{2}(n T)_{t}+S_{r}+\frac{k}{r} S-J \varphi_{r}+\frac{3}{2} n \frac{\left(T-T_{L}\right)}{\tau(T)}\right) & =0, \\
X^{[2]}\left(\lambda^{2}\left[\varphi_{r r}+\frac{k}{r} \varphi_{r}\right]+c(r)-n\right) & =0,
\end{aligned}
$$

whenever (2) is satisfied and where

$$
\mathrm{X}^{[2]}=\mathrm{X}+\zeta_{1}^{1} \frac{\partial}{\partial \mathrm{n}_{\mathrm{t}}}+\zeta_{2}^{1} \frac{\partial}{\partial \mathrm{n}_{\mathrm{r}}}+\zeta_{1}^{2} \frac{\partial}{\partial \mathrm{T}_{\mathrm{t}}}+\zeta_{2}^{2} \frac{\partial}{\partial \mathrm{T}_{\mathrm{r}}}+\zeta_{1}^{3} \frac{\partial}{\partial \varphi_{\mathrm{t}}}+\zeta_{2}^{3} \frac{\partial}{\partial \varphi_{\mathrm{r}}}+\zeta_{22}^{1} \frac{\partial}{\partial \mathrm{n}_{\mathrm{rr}}}+\zeta_{22}^{2} \frac{\partial}{\partial \mathrm{T}_{\mathrm{rr}}} .
$$

When expanded and separated, the determining equations (3) span many pages. Using the program Yalie [4] written in Mathematica, we obtain 38 equations. After simplifying the 38 determining equations we get the following equations (we only consider the case $\mathrm{k} \neq 0$, the case $\mathrm{k}=0$ was investigated in [10]).

$$
\begin{aligned}
& \xi^{1}=b_{1} t+b_{0}, \xi^{2}=\frac{r}{2}\left[a_{1} q+b_{1}\right], \eta^{1}=\left[a_{1}(1-q)-b_{1}\right] n, \eta^{2}=a_{1} T, \eta^{3}=a_{1} \varphi+F(t), \\
& \mu^{(1)}=\mu_{0}^{(1)} \mathrm{T}^{q^{-1}}, \mu^{(2)}=\mu_{0}^{(2)} \mathrm{T}^{\mathrm{q}-1}, \tau=\tau(T), c=c(r), \\
& a_{1} T\left(T_{L}-T\right) \tau_{T}+\left[b_{1}\left(T-T_{L}\right)+a_{1} T_{L}\right] \tau=0,2\left[b_{1}+a_{1}(q-1)\right] c+\left(b_{1}+a_{1} q\right) r_{r}=0 .
\end{aligned}
$$

Where $a_{1}, b_{1}, \mu_{0}^{(1)}, \mu_{0}^{(2)}$ are constants and $\mathrm{F}(\mathrm{t})$ is a constant function.

If we assume that $\mu^{(1)}(\mathrm{T}), \mu^{(2)}(\mathrm{T}), \tau(\mathrm{T})$ and $\mathrm{c}(\mathrm{r})$ are arbitrary functions of their arguments, we end up with the following symmetries

$$
\mathrm{X}_{1}=\frac{\partial}{\partial \mathrm{t}} \text { and } \mathrm{X}_{\mathrm{F}}=\mathrm{F}(\mathrm{t}) \frac{\partial}{\partial \varphi}
$$

where $\mathrm{F}(\mathrm{t})$ is a smooth function of $\mathrm{t}$. The symmetries $\mathrm{X}_{1}$ and $\mathrm{X}_{\mathrm{F}}$ span the so-called principal symmetry Lie algebra of (2).

Now our goal is to find specifications of the arbitrary elements that extend the principal Lie algebra. Consider equation $(6 b)$. If $b_{1}+a_{1} q=0$, then $b_{1}+a_{1}(q-1)=0$. Thus, $a_{1}=b_{1}=0$. This implies that $c(r)$ and $\tau(T)$ are arbitrary. We end up with the principal Lie algebra. As a result, this case does not lead to an extension. Therefore, we assume from now on that $b_{1}+a_{1} q \neq 0$. Solving equation in (6b), we obtain

$$
c(r)=c_{0} r^{\frac{-2\left(b_{1}+a_{1}-a_{1}\right)}{b_{1}+a_{19} q}}, c_{0} \text { constant. }
$$

If $\mathrm{a}_{1} \neq 0$, then

$$
\mathrm{c}(\mathrm{r})=\mathrm{c}_{0} \mathrm{r}^{\frac{-2(\mathrm{p}+\mathrm{q}-1)}{\mathrm{p}+\mathrm{q}}}, \text { provided } \mathrm{p}+\mathrm{q} \neq 0
$$

where $b_{1}=a_{1} p$. If $a_{1}=0$, then equation (6a) implies that $b_{1}=0$. i.e. we get the case $a_{1}=b_{1}=0$ which has already been dealt with. The solution to equation (6a) is

$$
\tau(T)=\tau_{0}\left(T-T_{L}\right) T^{p}, \tau_{0} \text { constant. }
$$

Thus, the extension of the principal symmetry Lie algebra is given by the operator

$$
X_{2}=p t \frac{\partial}{\partial t}+\frac{1}{2}(p+q) r \frac{\partial}{\partial r}+(1-p-q) n \frac{\partial}{\partial n}+T \frac{\partial}{\partial T}+\varphi \frac{\partial}{\partial \varphi} \text {. }
$$


Remark: For $\mathrm{q}=0$ and $\mathrm{q}=\frac{1}{2}$ we obtain the forms of $\mathbf{J}$ and $\mathbf{S}$ for Chen et al [3] model and Lyumkis et al [6] model respectively.

\subsection{The two-dimensional ET model for semiconductors}

Using the definition of the divergence and the Laplacian operators, the ET model system in two-dimensions becomes

$$
\begin{aligned}
\mathrm{n}_{\mathrm{t}}+\mathrm{J}_{\mathrm{x}}+\mathrm{J}_{\mathrm{y}} & =0, \\
\frac{3}{2}(\mathrm{nT})_{\mathrm{t}}+\mathrm{S}_{\mathrm{x}}+\mathrm{S}_{\mathrm{y}}-\mathrm{J}\left(\varphi_{\mathrm{x}}+\varphi_{\mathrm{y}}\right)+\frac{3}{2} \mathrm{n} \frac{\left(\mathrm{T}-\mathrm{T}_{\mathrm{L}}\right)}{\tau(\mathrm{T})} & =0, \\
\lambda^{2}\left(\varphi_{\mathrm{xx}}+\varphi_{\mathrm{yy}}\right)-\mathrm{n}+\mathrm{c}(\mathrm{x}, \mathrm{y}) & =0 .
\end{aligned}
$$

Employing the Yalie program [4] to generate the determining equations, we obtain 91 determining equations. After simplifications we get the following equations

$$
\begin{aligned}
& \xi^{1}=b_{0}-2 a_{1} t, \xi^{2}=a_{1} x+a_{0}, \xi^{3}=a_{1} y+a_{2}, \eta^{1}=\frac{2 a_{1}}{m}(2-m) n, \eta^{2}=\frac{4 a_{1}}{m} T, \\
& \eta^{3}=\frac{4 a_{1}}{m} \varphi+G(t), \mu^{(1)}=\mu_{0}^{(1)} T^{m-1}, \mu^{(2)}=\mu_{0}^{(2)} T^{m-1}, \tau=\tau(T), c=c(x, y), \\
& \tau\left[2 T_{L}+m\left(T_{L}-T\right)\right]+2 T\left(T_{L}-T\right) \tau_{T}=0, \\
& m\left[\left(a_{1} y+a_{2}\right) c_{y}+\left(a_{1} x+a_{0}\right) c_{x}\right]+2 a_{1}(m-2) c=0 .
\end{aligned}
$$

Where $\mathrm{a}_{0}, \mathrm{a}_{1}, \mathrm{a}_{2}, \mathrm{~b}_{0}, \mathrm{~m}, \mu_{0}^{(1)}$ and $\mu_{0}^{(2)}$ are constants and $\mathrm{G}(\mathrm{t})$ is a constant function.

If we assume that $\mu^{(\mathrm{i})}(\mathrm{T}), \tau(\mathrm{T})$ and $\mathrm{c}(\mathrm{x}, \mathrm{y})$ are arbitrary functions of their arguments, it can be shown that the principal symmetry Lie algebra of (7) is spanned by the operators

$$
X_{1}=\frac{\partial}{\partial t} \text { and } X_{G}=G(t) \frac{\partial}{\partial \varphi} \text {, where } G(t) \text { is a smooth function of } t .
$$

We now seek specifications of the arbitrary elements that extend the principal symmetry Lie algebra. The solution to equation (10) is

$$
\tau(\mathrm{T})=\tau_{0}\left(\mathrm{~T}-\mathrm{T}_{\mathrm{L}}\right) \mathrm{T}^{-\frac{2+\mathrm{m}}{2}}, \tau_{0} \text { constant }
$$

Next we consider equation (11) which is a first-order quasilinear PDE. If $\mathrm{a}_{1}=0$, then we have

$$
a_{2} c_{y}+a_{0} c_{x}=0 \text {, this implies that } c(x, y)=f\left(a_{2} x-a_{0} y\right) \text {, }
$$

where $\mathrm{f}$ is an arbitrary function of its argument. Suppose that $\mathrm{a}_{2} \neq 0$, then

$$
c(x, y)=f(x-\alpha y), \quad a_{0}=\alpha a_{2} .
$$

The extension of the symmetry Lie algebra is provided by

$$
\mathrm{X}_{2}=\alpha \frac{\partial}{\partial \mathrm{x}}+\frac{\partial}{\partial \mathrm{y}}
$$

If $\mathrm{a}_{2}=0$ and $\mathrm{a}_{0}=0$ and we have no extension of the principal symmetry Lie algebra. However, if $\mathrm{a}_{2}=0$ and $\mathrm{a}_{0} \neq 0$ we have $\mathrm{c}(\mathrm{x}, \mathrm{y})=\mathrm{g}(\mathrm{y})$, for an arbitrary function $\mathrm{g}$ of $\mathrm{y}$. This gives an extension 


$$
X_{2}=\frac{\partial}{\partial x}
$$

Suppose that $a_{1} \neq 0$. Equation (11) can then be expressed as

$$
(\mathrm{y}+\mathrm{q}) \mathrm{c}_{\mathrm{y}}+(\mathrm{x}+\mathrm{p}) \mathrm{c}_{\mathrm{x}}=\frac{2}{\mathrm{~m}}(2-\mathrm{m}) \mathrm{c}, \quad \mathrm{m} \neq 0,
$$

where $a_{2}=a_{1} q$ and $a_{0}=a_{1} p$. The solution of the above equation is

$$
c(x, y)=(y+q)^{\frac{2(2-m)}{m}} H\left(\frac{x+p}{y+q}\right)
$$

where $\mathrm{H}$ is an arbitrary function of its argument. Therefore, the extension of the principal Lie algebra is given by

$$
X_{2}=-2 t \frac{\partial}{\partial t}+(x+p) \frac{\partial}{\partial x}+(y+q) \frac{\partial}{\partial y}+\frac{2}{m}(2-m) n \frac{\partial}{\partial n}+\frac{4 T}{m} \frac{\partial}{\partial T}+\frac{4 \varphi}{m} \frac{\partial}{\partial \varphi} .
$$

Note that the case $\mathrm{m}=0$ does not yield an extension of the principal Lie algebra.

\section{SIMILARITY REDUCTIONS OF THE ET MODEL FOR SEMICONDUCTORS}

In many applications it is desirable to reduce partial differential equations (PDEs) to ordinary differential equations (ODEs), or at least reduce the number of independent variables. One of the procedures commonly used is dimensional analysis. Dimensional analysis is reminiscent of scaling symmetries and dimensionless variables are simply invariants of the scaling symmetry group [8]. By using symmetries more general than scaling symmetries it is possible to reduce the number of independent variables by introducing the invariants of these symmetries as new independent variables. Thus, the existence of symmetries for PDEs allows a sort of dimensional reduction. In this section we shall use symmetry methods to simplify our submodels whenever possible.

From section 3, we note that the symmetry structure of the submodels is a combination of a two-dimensional Lie algebra and an infinite-dimensional Lie algebra. In the following subsections we shall exploit mainly the finite-dimensional part of the symmetry Lie algebra. Precisely, using the notations of section 3, we shall look for solutions invariant under the operator $\mathrm{c}_{1} \mathrm{X}_{1}+\mathrm{c}_{2} \mathrm{X}_{2}$, where $\mathrm{c}_{1}$ and $\mathrm{c}_{2}$ are constants. This search can be splitted into two subcases $c_{2}=0$ and $c_{2} \neq 0$. These subcases amount to looking for solutions invariant under $X_{1}$ and $a X_{1}+X_{2}$, where $a$ is a constant. The first case (invariance under time translation) is trivial and will not be considered; as a result, for all the submodels, we shall concentrate on invariance under $\mathrm{aX}_{1}+\mathrm{X}_{2}$.

\subsection{The spherically symmetric case}

$$
\begin{aligned}
\mathrm{p}+\mathrm{q} & \neq 0, \quad \mu^{(1)}(\mathrm{T})=\mu_{0}^{(1)} \mathrm{T}^{\mathrm{q}-1}, \mu^{(2)}(\mathrm{T})=\mu_{0}^{(2)} \mathrm{T}^{\mathrm{q}-1}, \tau(\mathrm{T})=\tau_{0}\left(\mathrm{~T}-\mathrm{T}_{\mathrm{L}}\right) \mathrm{T}^{\mathrm{p}-1}, \\
\mathrm{c}(\mathrm{r}) & =\mathrm{c}_{0} \mathrm{r}^{\frac{2(1-\mathrm{p}-\mathrm{q})}{\mathrm{p}+\mathrm{q}}}, \mathrm{X}_{2}=\mathrm{pt} \frac{\partial}{\partial \mathrm{t}}+\frac{1}{2}(\mathrm{p}+\mathrm{q}) \mathrm{r} \frac{\partial}{\partial \mathrm{r}}+(1-\mathrm{p}-\mathrm{q}) \mathrm{n} \frac{\partial}{\partial \mathrm{n}}+\mathrm{T} \frac{\partial}{\partial \mathrm{T}}+\varphi \frac{\partial}{\partial \varphi} .
\end{aligned}
$$


The operator $X_{1}=\frac{\partial}{\partial t}$ is the same throughout. The characteristic equation for the invariants of $\mathrm{aX}+\mathrm{X}_{2}$ is

$$
\frac{\mathrm{dt}}{\mathrm{a}+\mathrm{pt}}=\frac{2}{(\mathrm{p}+\mathrm{q})} \frac{\mathrm{dr}}{\mathrm{r}}=\frac{1}{(1-\mathrm{p}-\mathrm{q})} \frac{\mathrm{dn}}{\mathrm{n}}=\frac{\mathrm{dT}}{\mathrm{T}}=\frac{\mathrm{d} \varphi}{\varphi} \text {. }
$$

The first equation of (12) prompts the consideration of the following subcases.

(i) $\quad \mathrm{p}=0$ : Equation (12) becomes

$$
\frac{\mathrm{dt}}{\mathrm{a}}=\frac{2}{\mathrm{q}} \frac{\mathrm{dr}}{\mathrm{r}}=\frac{1}{(1-\mathrm{q})} \frac{\mathrm{dn}}{\mathrm{n}}=\frac{\mathrm{dt}}{\mathrm{T}}=\frac{\mathrm{d} \varphi}{\varphi} .
$$

Thus, the invariant solutions assume the forms

$\mathrm{n}=v(\gamma) \mathrm{r}^{\frac{2(1-\mathrm{q})}{\mathrm{q}}}, \mathrm{T}=\omega(\gamma) \mathrm{r}^{\frac{2}{q}}$ and $\varphi=\chi(\gamma) \mathrm{r}^{\frac{2}{q}}$; where $\gamma=\mathrm{t}-\frac{2 \mathrm{a}(\ln \mathrm{r})}{\mathrm{q}}$ and $\nu, \omega, \chi$ are

arbitrary differentiable functions of $\gamma$. Substituting these invariant solutions into system (2) we get the reduced system

$$
\begin{aligned}
& \omega\left[4 a \mu_{0}^{(1)} \omega^{\mathrm{q}} v^{\prime}\left[\mathrm{a}\left(\chi^{\prime}-2 \mathrm{q} \omega^{\prime}\right)-\chi\right]+\omega\left\{\left[\mathrm{q}^{2}+8 \mathrm{a} \mu_{0}^{(1)} \omega^{\mathrm{q}}+2 \mathrm{a}(\mathrm{k}-1) \mathrm{q} \mu_{0}^{(1)} \omega^{\mathrm{q}}\right] v^{\prime}\right.\right. \\
& \left.\left.-4 \mathrm{a}^{2} \mu_{0}^{(1)} \omega^{\mathrm{q}} v^{\prime \prime}\right\}\right]+2 \mu_{0}^{(1)} \omega^{\mathrm{q}} v\left[(\mathrm{q}-\mathrm{kq}-2) \omega^{2}+\chi\left\{[2+(\mathrm{k}-1) \mathrm{q}] \omega-2 \mathrm{a}(\mathrm{q}-1) \omega^{\prime}\right\}\right. \\
& -2 \mathrm{a}^{2} \omega^{\prime}(\mathrm{q}-1)\left(\mathrm{q} \omega^{\prime}-\chi^{\prime}\right)+\mathrm{a} \omega\left[(\mathrm{q}-\mathrm{kq}-4) \chi^{\prime}+\mathrm{q}[4+(\mathrm{k}-1) \mathrm{q}] \omega^{\prime}+2 \mathrm{a}\left(\chi^{\prime \prime}-\mathrm{q} \omega^{\prime \prime}\right)\right]=0, \\
& \tau_{0} \omega\left\{8 \mathrm{a} \omega^{\mathrm{q}} v^{\prime}\left[-\left(\mu_{0}^{(1)}+\mu_{0}^{(1)}\right) \chi+\mathrm{a}\left[\left(\mu_{0}^{(1)}+\mu_{0}^{(2)}\right) \chi^{\prime}-2(\mathrm{q}+1) \mu_{0}^{(2)} \omega^{\prime}\right]\right]\right. \\
& \left.+\omega\left[\left(3 \mathrm{q}^{2}+32 \mathrm{a} \mu_{0}^{(2)} \omega^{\mathrm{q}}+4 \mathrm{aq}(\mathrm{k}-1) \mu_{0}^{(2)} \omega^{\mathrm{q}} v^{\prime}\right)-8 \mathrm{a}^{2} \mu_{0}^{(2)} \omega^{\mathrm{q}} v^{\prime \prime}\right]\right\} \\
& +v\left\{\omega^{2}\left(3 \mathrm{q}^{2}-32 \mu_{0}^{(2)} \tau_{0} \omega^{\mathrm{q}}-8 \mathrm{q}(\mathrm{k}-1) \mu_{0}^{(2)} \tau_{0} \omega^{\mathrm{q}}\right)+v\left\{\omega ^ { 2 } \left(3 \mathrm{q}^{2}-32 \mu_{0}^{(2)} \tau_{0} \omega^{\mathrm{q}}\right.\right.\right. \\
& \left.-8 \mathrm{q}(\mathrm{k}-1) \mu_{0}^{(2)} \tau_{0} \omega^{\mathrm{q}}\right)-8 \tau_{0} \omega^{\mathrm{q}}\left(\mu_{0}^{(1)} \chi^{2}+\mathrm{a} \chi\left[\mathrm{q}\left(\mu_{0}^{(1)}+\mu_{0}^{(2)}\right) \omega^{\prime}-2 \mu_{0}^{(1)} \chi^{\prime}\right]\right. \\
& \left.+\mathrm{a}^{2}\left[\mu_{0}^{(1)} \chi^{\prime 2}-\mathrm{q}\left(\mu_{0}^{(1)}+\mu_{0}^{(2)}\right) \chi^{\prime} \omega^{\prime}+\mathrm{q}(\mathrm{q}+1) \mu_{0}^{(2)} \omega^{\prime 2}\right]\right)+\tau_{0} \omega\left[4 \left(2 \mu_{0}^{(1)}+[4+\right.\right. \\
& \left.(\mathrm{k}-1) \mathrm{q}] \mu_{0}^{(2)}\right) \chi \omega^{\mathrm{q}}+4 \mathrm{a}\left(2 \mu_{0}^{(1)}+[6+(\mathrm{k}-1) \mathrm{q}] \mu_{0}^{(2)}\right) \chi^{\prime} \omega^{\mathrm{q}}+3 \mathrm{q}^{2} \omega^{\prime}+32 \mathrm{a} \mu_{0}^{(2)} \omega^{\mathrm{q}} \omega^{\prime} \\
& +28 \mathrm{aq} \mu_{0}^{(2)} \omega^{\mathrm{q}} \omega^{\prime}+4 \mathrm{akq} \mu_{0}^{(2)} \omega^{\mathrm{q}} \omega^{\prime}-4 \mathrm{aq} \mu_{0}^{2} \omega \omega^{\mathrm{q}} \omega^{\prime \prime}+4 \mathrm{akq} \mu_{0}^{2(2)} \omega^{\mathrm{q}} \omega^{\prime}+8 \mathrm{a}^{2} \mu_{0}^{(2)} \omega^{\mathrm{q}} \chi^{\prime \prime} \\
& \left.\left.\quad-8 \mathrm{a}^{2} \mu_{0}^{(2)} \omega^{\mathrm{q}} \omega^{\prime \prime}-8 \mathrm{a}^{2} \mathrm{q} \mu_{0}^{(2)} \omega^{\mathrm{q}} \omega^{\prime \prime}\right]\right\}=0, \\
& 4 \mathrm{a}^{2} \lambda^{2} \chi^{\prime \prime}+2 \mathrm{a} \lambda^{2}[\mathrm{q}(1-\mathrm{k})-4] \chi^{\prime}+2 \lambda^{2}[2+(\mathrm{k}-1) \mathrm{q}] \chi-\mathrm{q}^{2} v+\mathrm{q}^{2} \mathrm{c}_{0}=0 .
\end{aligned}
$$

In the above system and the subsequent systems a prime denotes differentiation with respect to the similarity variable $\gamma$.

(ii) $\quad \mathrm{p} \neq 0$ : The invariant solutions read

$\mathrm{n}=v(\gamma)(\mathrm{a}+\mathrm{pt})^{\frac{1-\mathrm{p}-\mathrm{q}}{\mathrm{p}}}, \mathrm{T}=\omega(\gamma)(\mathrm{a}+\mathrm{pt})^{\frac{1}{\mathrm{p}}}$ and $\varphi=\chi(\gamma)(\mathrm{a}+\mathrm{pt})^{\frac{1}{\mathrm{p}}}$;

where $\gamma=\mathrm{r}(\mathrm{a}+\mathrm{pt})^{-\frac{(p+q)}{2 \mathrm{p}}}, v, \omega$ and $\chi$ are differentiable functions of their arguments. The substitution of equations (16) into system (2) gives the following reduced system $\omega\left\{2 \gamma \mu_{0}^{(1)} \omega^{\mathrm{q}} v^{\prime}\left(2 \mathrm{q} \omega^{\prime}-\chi^{\prime}\right)-\omega-\omega\left[\left(\mathrm{p} \gamma^{2}+\mathrm{q} \gamma^{2}+2 \mathrm{k} \mu_{0}^{(1)} \omega^{\mathrm{q}}\right) \nu^{\prime}+2 \gamma \mu_{0}^{(1)} \omega^{\mathrm{q}} v^{\prime \prime}\right)\right\}$ $+2 v\left[(\mathrm{p}+\mathrm{q}-1) \gamma \omega^{2}+\gamma \mu_{0}^{(1)} \omega^{\mathrm{q}} \omega^{\prime}(\mathrm{q}-1)\left(\mathrm{q} \omega^{\prime}-\chi^{\prime}\right)-\mu_{0}^{(1)} \omega^{\mathrm{q}+1}\left(\mathrm{k} \chi^{\prime}-\mathrm{kq} \omega^{\prime}+\gamma \chi^{\prime \prime}-\mathrm{q} \gamma \omega^{\prime \prime}\right)=0,(17)\right.$ $\tau_{0} \omega\left[4 \gamma \omega^{\mathrm{q}} v^{\prime}\left[2(\mathrm{q}+1) \mu_{0}^{(2)} \omega^{\prime}-\left(\mu_{0}^{(1)}+\mu_{0}^{(2)}\right) \chi^{\prime}\right]-\left\{\left[3 \mathrm{p} \gamma^{2}+3 \mathrm{q} \gamma^{2}+4 \mathrm{k} \mu_{0}^{(2)} \omega^{\mathrm{q}}\right] v^{\prime}\right.\right.$ $\left.\left.+4 \mathrm{k} \gamma \mu_{0}^{(2)} \omega^{\mathrm{q}} v^{\prime \prime}\right\}\right]+v\left\{6 \gamma \gamma^{2}\left[1-(\mathrm{q}-2) \tau_{0} \omega^{\mathrm{p}}-\mathrm{p} \tau_{0}\right]-4 \gamma \tau_{0} \omega^{\mathrm{p}+\mathrm{q}}\left[\mu_{0}^{(1)} \chi^{2}\right.\right.$ 


$$
\begin{aligned}
& \left.-\left(\mu_{0}^{(1)}+\mu_{0}^{(2)}\right) \chi^{\prime} \omega^{\prime}+\mathrm{q}(\mathrm{q}+1) \mu_{0}^{(2)} \omega^{\prime 2}\right]+\tau_{0} \omega^{\mathrm{p}+1}\left[4 \mathrm{k} \mu_{0}^{(2)} \omega^{\mathrm{q}} \chi^{\prime}-\left(3 \mathrm{p} \gamma^{2}+3 \mathrm{q} \gamma^{2}\right.\right. \\
& \left.\left.\left.+4 \mathrm{k}(\mathrm{q}+1) \mu_{0}^{(2)} \omega^{\mathrm{q}}\right) \omega^{\prime}+4 \gamma \mu_{0}^{(2)} \omega^{\mathrm{q}}\left(\chi^{\prime \prime}-(\mathrm{q}+1) \omega^{\prime \prime}\right)\right]\right\}=0 \text {, } \\
& \gamma^{2} \lambda^{2} \chi^{\prime \prime}+\mathrm{k} \gamma \lambda^{2} \chi^{\prime}-\gamma^{2} v+\mathrm{c}_{0} \gamma^{[2 /(\mathrm{p}+\mathrm{q})]}=0 \text {. }
\end{aligned}
$$

\subsection{The two-dimensional case}

(i) $\mu^{(1)}(\mathrm{T})=\mu_{0}^{(1)} \mathrm{T}^{\mathrm{m}-1}, \mu^{(2)}(\mathrm{T})=\mu_{0}^{(2)} \mathrm{T}^{\mathrm{m}-1}, \tau(\mathrm{T})=\tau_{0}\left(\mathrm{~T}-\mathrm{T}_{\mathrm{L}}\right) \mathrm{T}^{-\frac{2+\mathrm{m}}{2}}, \mathrm{c}(\mathrm{x}, \mathrm{y})=\mathrm{g}(\mathrm{y})$,

and $X_{2}=\frac{\partial}{\partial x}$. The characteristic equation for the invariants of $a X_{1}+X_{2}$ is

$$
\frac{\mathrm{dt}}{\mathrm{a}}=\frac{\mathrm{dx}}{1}=\frac{\mathrm{dy}}{0}=\frac{\mathrm{dn}}{0}=\frac{\mathrm{dT}}{0}=\frac{\mathrm{d} \varphi}{0} .
$$

This gives the similarity variables

$$
\gamma=\mathrm{t}-\mathrm{ax}, \beta=\mathrm{y}
$$

and the similarity solutions

$$
\mathrm{n}=v(\gamma, \beta), \mathrm{T}=\omega(\gamma, \beta), \varphi=\chi(\gamma, \beta),
$$

where $v, \omega$ and $\chi$ are arbitrary functions of $\gamma$ and $\beta$. The equations (20) are substituted into system (7) to give the following reduced system

$$
\begin{aligned}
& \omega^{2} v_{\gamma}+(\mathrm{m}-1) \mu_{0}^{(1)} v \omega^{\mathrm{m}}\left[\chi_{\beta} \omega_{\beta}-\mathrm{m} \omega^{2}{ }_{\beta}+\mathrm{a}^{2} \omega_{\gamma}\left(\chi_{\gamma}-\mathrm{m} \omega_{\gamma}\right)\right]-\mu_{0}^{(1)} \omega^{\mathrm{m}+2}\left(v_{\beta \beta}+\mathrm{a}^{2} v_{\gamma \gamma}\right) \\
& +\mu_{0}^{(1)} \omega^{\mathrm{m}+1}\left\{v_{\beta}\left(\chi_{\beta}-2 \mathrm{~m} \omega_{\beta}\right)+\mathrm{a}^{2} v_{\gamma}\left(v_{\gamma}+2 \mathrm{~m} \omega_{\gamma}\right)+v\left[\chi_{\beta \beta}-\mathrm{m} \omega_{\beta \beta}+\mathrm{a}^{2}\left(\chi_{\gamma \gamma}-\mathrm{m} \omega_{\gamma \gamma}\right)\right]\right\}=0,(21) \\
& \frac{1}{2 \tau_{0} \omega}\left\{\tau_{0} \omega\left[3 \omega v_{\gamma}+2 \omega^{\mathrm{m}}\left(v_{\beta} \chi_{\beta}+\mathrm{a}^{2} v_{\gamma} \chi_{\gamma}\right)\left(\mu_{0}^{(1)}+\mu^{(2)}{ }_{0}\right)\right]+v\left[3 \omega^{[(\mathrm{m}+4) / 2]}+3 \tau_{0} \omega \omega_{\gamma}\right.\right. \\
& \left.\left.+2 \mathrm{~m} \tau_{0} \omega^{\mathrm{m}}\left(\chi_{\beta} \omega_{\beta}+\mathrm{a}^{2} \chi_{\gamma} \omega_{\gamma}\right)\left(\mu_{0}^{(1)}+\mu^{(2)}\right)+2 \mu^{(2)}{ }_{0} \tau_{0} \omega^{\mathrm{m}+1}\left(\chi_{\beta \beta}+\mathrm{a}^{2} \chi_{\gamma \gamma}\right)\right]\right\} \\
& -\mu^{(1)}{ }_{0}{ }^{2} \omega^{2 \mathrm{~m}-4}\left[(\mathrm{~m}-1) v \chi_{\beta} \omega_{\beta}+\omega\left(v_{\beta} \chi_{\beta}+v \chi_{\beta \beta}\right)\right]^{2}+\mu^{(2)}{ }_{0} \omega^{\mathrm{m}-1}\left[\mathrm{~m}(\mathrm{~m}+1) v \omega^{2}{ }_{\beta}+\omega^{2} v_{\beta \beta}\right. \\
& \left.+\omega(\mathrm{m}+1)\left(2 v_{\beta} \omega_{\beta}+v \omega_{\beta \beta}\right)\right]+\mathrm{a}^{4} \mu_{0}^{(1)} \omega^{2 \mathrm{~m}-4}\left[(\mathrm{~m}-1) v \chi_{\gamma} \omega_{\gamma}+\omega\left(v_{\gamma} \chi_{\gamma}+v \chi_{\gamma \gamma}\right)\right]^{2} \\
& +\mathrm{a}^{2} \mu_{0}^{(1)} \omega^{\mathrm{m}-1}\left[\mathrm{~m}(\mathrm{~m}+1) v \omega_{\gamma}{ }_{\gamma}+\omega^{2} v_{\gamma \gamma}+\omega(\mathrm{m}+1)\left(2 v_{\gamma} \omega_{\gamma}+v \omega_{\gamma \gamma}\right)\right]=0,(22) \\
& \lambda^{2}\left(\chi_{\beta \beta}+\mathrm{a}^{2} \chi_{\gamma \gamma}\right)-v+\mathrm{g}(\beta)=0 .(23)
\end{aligned}
$$

Here and thereafter the subscripts on $v, \omega$ and $\chi$ denote partial differentiation.

(ii) $\mu^{(1)}(\mathrm{T})=\mu_{0}^{(1)} \mathrm{T}^{\mathrm{m}-1}, \mu^{(2)}(\mathrm{T})=\mu_{0}^{(2)} \mathrm{T}^{\mathrm{m}-1}, \tau(\mathrm{T})=\tau_{0}\left(\mathrm{~T}-\mathrm{T}_{\mathrm{L}}\right) \mathrm{T}^{-[(2+\mathrm{m}) / 2]}, \mathrm{c}(\mathrm{x}, \mathrm{y})=\mathrm{f}(\mathrm{x}-\alpha \mathrm{y})$, and $\mathrm{X}_{2}=\alpha \frac{\partial}{\partial \mathrm{x}}+\frac{\partial}{\partial \mathrm{y}}$. The linear combination $\mathrm{aX}+\mathrm{X}_{2}$ gives the characteristic system

$$
\frac{\mathrm{dt}}{\mathrm{a}}=\frac{\mathrm{dx}}{\alpha}=\frac{\mathrm{dy}}{1}=\frac{\mathrm{dn}}{0}=\frac{\mathrm{dT}}{0}=\frac{\mathrm{d} \varphi}{0} .
$$

The similarity solutions are

$$
\mathrm{n}=v(\gamma, \beta), \mathrm{T}=\omega(\gamma, \beta), \varphi=\chi(\gamma, \beta)
$$

where $\gamma=\alpha \mathrm{t}-\mathrm{ax}, \beta=\mathrm{x}-\alpha \mathrm{y}$ and $v, \omega$ and $\chi$ are differentiable functions of their arguments. The substitution of (24) into system (7) gives

$$
\begin{aligned}
& \alpha \omega^{2} v_{\gamma}-(\mathrm{m}-1) \mu_{0}^{(1)} v \omega^{\mathrm{m}}\left[\left(1+\alpha^{2}\right)\left(\mathrm{m} \omega_{\beta}-\chi_{\beta}\right) \omega_{\beta}+\mathrm{a} \omega_{\gamma} \chi_{\beta}+\mathrm{a} \omega_{\beta}\left(\chi_{\gamma}-2 \mathrm{~m} \omega_{\gamma}\right)\right. \\
& \left.+\mathrm{a}^{2} \omega_{\gamma}\left(\mathrm{m} \omega_{\gamma}-\chi_{\gamma}\right)\right]-\mu_{0}^{(1)} \omega^{\mathrm{m}+2}\left[\left(1+\alpha^{2}\right) v_{\beta \beta}+\mathrm{a}\left(\mathrm{a} v_{\gamma \gamma}-2 v_{\gamma \beta}\right)\right]-\mu_{0}^{(1)} \omega^{\mathrm{m}+1}\left\{v _ { \beta } \left[\left(1+\alpha^{2}\right)\right.\right.
\end{aligned}
$$




$$
\begin{aligned}
& \left.\times\left(\chi_{\beta}-2 \mathrm{~m} \omega_{\beta}\right)-\mathrm{a}\left(\chi_{\gamma}-2 \mathrm{~m} \omega_{\gamma}\right)\right]+\mathrm{a} v_{\gamma}\left[2 \mathrm{~m} \omega_{\beta}-\chi_{\beta}+\mathrm{a}\left(\chi_{\gamma}-2 \mathrm{~m} \omega_{\gamma}\right)\right]+v\left[\left(1+\alpha^{2}\right)\right. \\
& \left.\left.\times\left(\chi_{\beta \beta}-\mathrm{m} \omega_{\beta \beta}\right)+\mathrm{a}\left(2 \mathrm{~m} \omega_{\gamma \beta}-2 \chi_{\gamma \beta}+\mathrm{a} \chi_{\gamma \gamma}-\mathrm{am} \omega_{\gamma \gamma}\right)\right]\right\}=0,(25) \\
& \frac{1}{2 \tau_{0} \omega}\left\{\tau _ { 0 } \omega \left(3 \alpha \omega v_{\gamma}+2 \omega^{\mathrm{m}}\left[\mathrm{a} \mu_{0}^{(2)} v_{\gamma}\left(\mathrm{a} \chi_{\gamma}-\chi_{\beta}\right)+v_{\beta}\left\{\left[\mu_{0}^{(2)}\left(1-\mathrm{a} \mu_{0}^{(2)} \chi_{\gamma}\right)\right.\right.\right.\right.\right. \\
& \left.\left.\left.\left.+\alpha^{2}\left(\mu_{0}^{(1)}+\mu_{0}^{(2)}\right)\right] \chi_{\beta}\right\}\right]\right)+v\left(3 \omega^{[(m+4) / 2]}+3 \alpha \tau_{0} \omega \omega_{\gamma}+2 \mathrm{~m} \tau_{0} \omega^{\mathrm{m}}\left[\mathrm{a} \mu_{0}^{(2)} \chi_{\gamma}\left(\mathrm{a} \omega_{\gamma}-\omega_{\beta}\right)\right.\right. \\
& \left.+\chi_{\beta}\left\{\left[\mu_{0}^{(2)}+\alpha^{2}\left(\mu_{0}^{(1)}+\mu_{0}^{(2)}\right)\right] \omega_{\beta}-\mathrm{a} \mu_{0}^{(2)} \omega_{\gamma}\right\}\right]+2 \mu_{0}^{(2)} \tau_{0} \omega^{\mathrm{m}+1}\left[\left(1+\alpha^{2}\right) \chi_{\beta \beta}\right. \\
& \left.\left.\left.+\mathrm{a}\left(\mathrm{a} \chi_{\gamma \gamma}-2 \chi_{\gamma \beta}\right)\right]\right)\right\}-\alpha^{2} \mu_{0}^{(1) 2} \omega^{2 \mathrm{~m}-4}\left[(\mathrm{~m}-1) \nu_{\beta} \omega_{\beta}+\omega\left(v_{\beta} \chi_{\beta}+v \chi_{\beta \beta}\right)\right]^{2} \\
& +\omega^{\mathrm{m}-1}\left\{\alpha \mu_{0}^{(2)}(\mathrm{m}+1)\left[\operatorname{mv} \omega_{\beta}^{2}+\omega\left(2 v_{\beta} \omega_{\beta}+v \omega_{\beta \beta}\right)+\omega^{2} v_{\beta \beta}\right]\right\} \\
& -\omega^{\mathrm{m}-1}\left\{\mu_{0}^{(1)}\left(\chi_{\beta}-\mathrm{a} \chi_{\gamma}\right)\left[\omega\left(\mathrm{a} v_{\gamma}-v_{\beta}\right)+\mathrm{m} v\left(\mathrm{a} \omega_{\gamma}-\omega_{\beta}\right)\right]\right\}-\mu_{0}^{(1) 2} \omega^{2 \mathrm{~m}-4}[v(\mathrm{~m}-1) \\
& \left.\times\left(\chi_{\beta}-\mathrm{a} \chi_{\gamma}\right)\left(\omega_{\beta}-\mathrm{a} \omega_{\gamma}\right)+\omega\left[\left(v_{\beta}-\mathrm{a} v_{\gamma}\right)\left(\chi_{\beta}-\mathrm{a} \chi_{\gamma}\right)+v\left(\chi_{\beta \beta}-2 \mathrm{a} \chi_{\gamma \beta}+\mathrm{a}^{2} \chi_{\gamma \gamma}\right)\right]\right]^{2} \\
& -\mu_{0}^{(2)}\left\{2 \omega^{\mathrm{m}}(\mathrm{m}+1)\left(v_{\beta}-\mathrm{a} v_{\gamma}\right)\left(\omega_{\beta}-\mathrm{a} \omega_{\gamma}\right)+\omega^{\mathrm{m}+1}\left[v_{\beta \beta}+\mathrm{a}\left(\mathrm{a} v_{\gamma \gamma}-2 v_{\gamma \beta}\right)\right]+v(\mathrm{~m}+1)\right. \\
& \left.\times\left[\omega^{\mathrm{m}} \omega_{\beta \beta}+\mathrm{m} \omega^{\mathrm{m}-1}\left(\omega_{\beta}-\mathrm{a} \omega_{\gamma}\right)^{2}+\mathrm{a} \omega^{\mathrm{m}}\left(\mathrm{a} \omega_{\gamma \gamma}-2 \omega_{\gamma \beta}\right)\right]\right\}=0, \\
& \lambda^{2}\left[\left(1+\alpha^{2}\right) \chi_{\beta \beta}+\mathrm{a}\left(\mathrm{a} \chi_{\gamma \gamma}-2 \chi_{\gamma \beta}\right)\right]-v+\mathrm{f}(\beta)=0 . \\
& \mathrm{m} \neq 0,2, \quad \mu^{(1)}(\mathrm{T})=\mu_{0}^{(1)} \mathrm{T}^{\mathrm{m}-1}, \quad \mu^{(2)}(\mathrm{T})=\mu_{0}^{(2)} \mathrm{T}^{\mathrm{m}-1}, \\
& \tau(\mathrm{T})=\tau_{0}\left(\mathrm{~T}-\mathrm{T}_{\mathrm{L}}\right) \mathrm{T}^{-\frac{2+\mathrm{m}}{2}}, \quad \mathrm{c}(\mathrm{x}, \mathrm{y})=(\mathrm{y}+\mathrm{q})^{\frac{2(2-\mathrm{m})}{\mathrm{m}}} \mathrm{H}\left(\frac{\mathrm{x}+\mathrm{p}}{\mathrm{y}+\mathrm{q}}\right) \\
& X_{2}=-2 t \frac{\partial}{\partial t}+(x+p) \frac{\partial}{\partial x}+(y+q) \frac{\partial}{\partial y}+\frac{2}{m}(2-m) n \frac{\partial}{\partial n}+\frac{4 T}{m} \frac{\partial}{\partial T}+\frac{4 \varphi}{m} \frac{\partial}{\partial \varphi} .
\end{aligned}
$$

The characteristic equation corresponding to $a X_{1}+X_{2}$ is

$$
\frac{d t}{a-2 t}=\frac{d x}{x+p}=\frac{d y}{y+q}=\frac{m}{2(2-m)} \frac{d n}{n}=\frac{m}{4} \frac{d T}{T}=\frac{m}{4} \frac{d \varphi}{\varphi} .
$$

Thus we obtain the invariant solutions

$$
\mathrm{n}=v(\gamma, \beta)(\mathrm{x}+\mathrm{p})^{\frac{2(2-\mathrm{m})}{\mathrm{m}}}, \mathrm{T}=\omega(\gamma, \beta)(\mathrm{x}+\mathrm{p})^{\frac{4}{\mathrm{~m}}} \text { and } \varphi=\chi(\gamma, \beta)(\mathrm{x}+\mathrm{p})^{\frac{4}{\mathrm{~m}}}
$$

where $\gamma=(a-2 t)(x+p)^{2}, \beta=\frac{x+p}{y+p}$ and $v, \omega$ and $\chi$ are arbitrary functions of their arguments. When equations (28) are substituted into system (7) we obtain a system of PDEs that spans many pages. The interested reader can use Mathematica or any package for symbolic computation to obtain the reduced system.

\section{CONCLUSION}

In this paper, we have performed a complete Lie symmetry classification of the spherically symmetric and two-dimensional ET models for semiconductor. i.e we obtained all the forms of the arbitrary elements (energy relaxation time, carrier mobility and the doping profile) that maximize the symmetry Lie algebra. 
Following the usual modus operandi in symmetry analysis $[2,5,7,8]$, we exploited the symmetries of the submodels to perform similarity reductions. The reduced submodels are still highly nonlinear and hence, difficult to solve analytically. The next logical step of this work will be a numerical investigation of the reduced submodels. This investigation might be of great importance in the simulation and design of semiconductors.

Finally it might be important to investigate the full $1+3$ model without a priori symmetry assumptions (spherical symmetry for instance). It might not be a simple task as the classifying relations can be very difficult to analyse. If this happens, the so-called method of preliminary group classification may be used.

Acknowledgements- We would like to thank Prof F M Mahomed for his comments and suggestions while proof-reading this work. M Molati acknowledges with gratitude financial support from the Lesotho Government through N.M.D.S. The authors also thank Mrs Nkabinde T E for typing part of the manuscript.

\section{REFERENCES}

1. N. B. Abdallah and P. Degond, On a hierarchy of macroscopic models for semiconductors, Journal of Mathematical Physics 37 205-31, 1996.

2. G. W. Bluman and S. Kumei, Symmetries and Differential Equations, SpringerVerlag, New York, 1989.

3. D. Chen et al, An improved energy-transport model including nonparabolicity and non-Maxwellian distribution effects, IEEE Electron Device Letter 13 26-28, 1992.

4. J. M. Díaz, Short guide to Yalie package for Lie symmetries, http://www.mathsource.com/MathSource/Applications/Mathematics/ 0211-778/ YaLie.ps, 2000.

5. N. H. Ibragimov, Elementary Lie Group Analysis and Ordinary Differential equations, John Wiley, Chichester, 1999.

6. E. Lyumkis et al, Transient semiconductor device simulation including energy balance equation, Int. J. Comput. Math. Electron. Eng. 11, 311-325, 1992.

7. P. J. Olver, Applications of Lie Groups to Differential Equations SpringerVerlag, New York, 1986.

8. L. V. Ovsiannikov, Group Analysis of Differential Equations Academic Press, New York, 1982

9. R. F. Pierret, Semiconductor fundamentals, Vol. 1, Addison-Wesley, Massachusetts, 1988.

10. V. Romano and A. Valenti, Symmetry Analysis and Exact Invariant Solutions for a Class of Energy-Transport Models of Semiconductors, Journal of Physics A: Mathematical and General 35, 1751-1762, 2002.

11. S. M. Sze, Semiconductor Devices, Physics and Technology, John Wiley, New York, 1985. 